\title{
Detection of Serous Tubal Intraepithelial Carcinoma along with High Grade Serous Carcinoma of Both Ovaries
}

\author{
Hina Tariq \\ Department of Histopathology, Army Medical College, Rawalpindi, Pakistan
}

\begin{abstract}
High-grade serous carcinoma (HGSC) of ovary is the commonest of all ovarian malignant tumours and is associated with high mortality rate. Serous tubal intraepithelial carcinoma (STIC) is believed to be the precursor lesion. In literature, only $40-50 \%$ of HGSC shows concurrent STIC, even after thorough sampling. We, herein, present a case of a 40-year female, who underwent staging laparotomy for bilateral ovarian carcinoma. She was diagnosed as HGSC of both ovaries. Left ovary capsule was focally ruptured. Peritoneal washings showed metastatic carcinoma. Provisional FIGO stage was IC. During sampling, concurrent finding of STIC was documented in right fallopian tube. No invasive carcinoma was present in both entirely submitted fallopian tubes.
\end{abstract}

Key Words: High grade serous carcinoma, Ovary, Serous tubal intraepithelial carcinoma, TP53.

How to cite this article: Tariq H. Detection of Serous Tubal Intraepithelial Carcinoma along with High Grade Serous Carcinoma of Both Ovaries. J Coll Physicians Surg Pak 2021; 31(09):1108-1110.

\section{INTRODUCTION}

High-grade serous carcinoma (HGSC) makes 50 - $60 \%$ of all ovarian malignant tumours. Conventionally, surface epithelium of the ovary was thought to be the origin of $\mathrm{HGSC}^{1}{ }^{1}$ More recently, serous tubal intraepithelial carcinoma (STIC) was suggested as the probable non-invasive precursor lesion. ${ }^{1,2}$ About $90 \%$ of HGSCs arise sporadically and in these cases, the significance of STIC is still not clear. ${ }^{1}$

Classical STIC shows pleomorphism, hyperchromasia, increased nuclear to cytoplasmic ratio, lack of ciliated cells, loss of polarity with or without epithelial stratification, and occasional mitotic figures. Majority of these cases reveal strong p53 immunostaining and demonstrate p53 mutations. These findings together are known as "p53 signature". 1

We, herein, present a case of a 40-year female, who underwent staging laparotomy for bilateral ovarian carcinoma. During sampling, concurrent finding of STIC was documented in right fallopian tube. No invasive carcinoma was present in both entirely submitted fallopian tubes.

\section{CASE REPORT}

A 40-year female underwent staging laparotomy for HGSC of both ovaries. Specimens included total abdominal hysterectomy with bilateral salpingo-oophorectomy, omentectomy and peritoneal washing. Both ovaries were enlarged and involved by tumour.

Correspondence to: Dr. Hina Tariq, Department of Histopathology, Army Medical College, Rawalpindi, Pakistan E-mail:dr.hina2013@gmail.com

Received: November 11, 2019; Revised: December 22, 2019; Accepted: February 10, 2020

DOI: https://doi.org/10.29271/jcpsp.2021.09.1108
Surfaces of both ovaries were not involved by tumour. Left ovary showed focally ruptured capsule. Fallopian tubes, uterus and omentum were grossly unremarkable.

Histological examination of both ovaries revealed branching papillary fronds, complex glandular structures, marked pleomorphism, prominent nucleoli, stratification and frequent mitoses. It was diagnosed as HGSC, involving both ovaries. No invasive carcinoma was found in both fallopian tubes. Uterus including uterine serosa, cervix, parametrium and omentum did not show any evidence of carcinoma. Peritoneal washings showed metastatic carcinoma, of primary HGSC of ovaries. Provisional FIGO stage was IC.

Multifocal STIC was identified at the fimbriated end of right fallopian tube. Left fallopian tube was unremarkable. Both fallopian tubes were entirely submitted. However, no invasive carcinoma was found.

Immunohistochemistry (IHC) panel was applied on HGSC and STIC. HGSC showed strong diffuse positivity for CK7, WT1; while it was negative for CK20. Both showed strong diffuse expression of p53 (TP53 Missense Mutant). Proliferative index (Ki-67) was $40-50 \%$ in STIC (Figures 1 and 2 ).

\section{DISCUSSION}

According to WHO, more than 2 million cases of ovarian carcinoma are reported per year and about 1.4 million per year die of this lethal disease. ${ }^{3}$ Approximately, $90 \%$ of ovarian tumours are of surface epithelial origin; and among them, $70 \%$ are serous carcinomas. Serous carcinomas are further subdivided into high grade and low grade types. Both these types are basically two different diseases and have different pathogenesis. ${ }^{4}$ 


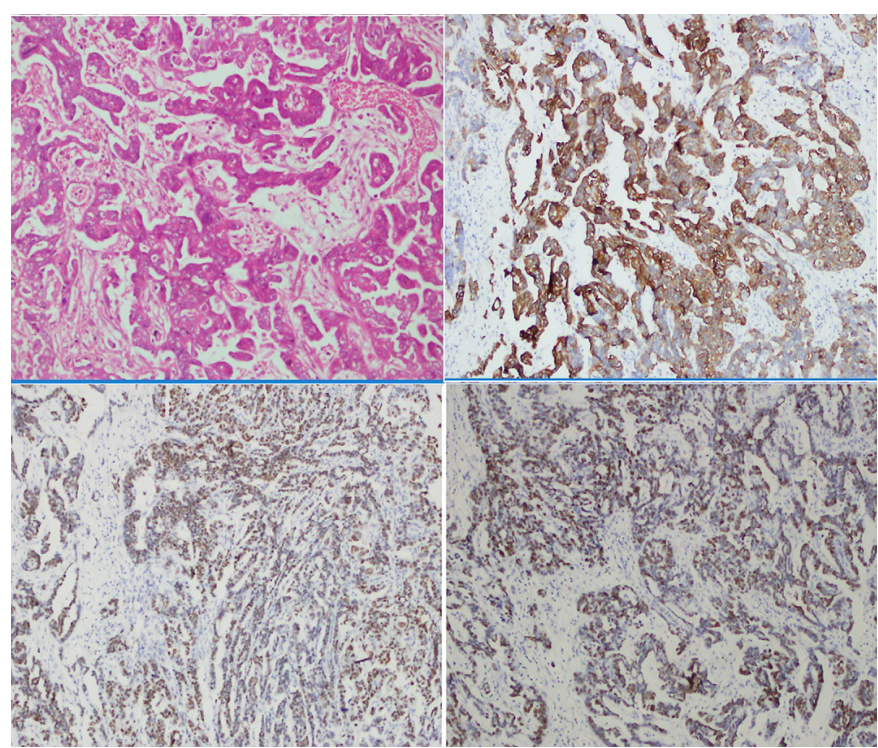

Figure 1: High grade serous carcinoma of ovary. (A). H \& E (20X) (B). Diffuse cystoplasmic staining pattern of CK7 (20X) (C). Diffuse nuclear staining of WT1 (20X) (D). Strong diffuse staining of p53 (Mutant expression) 20X.

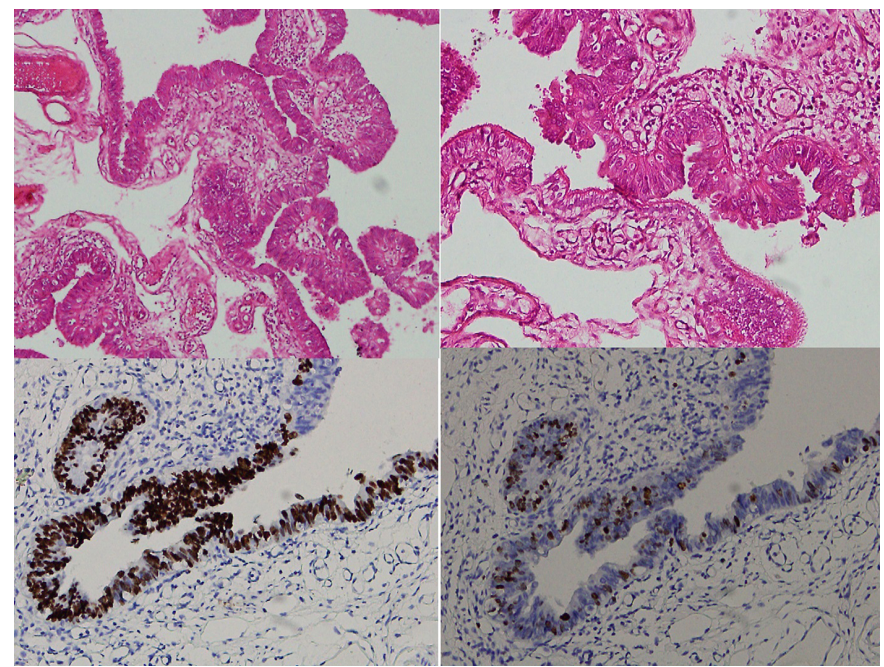

Figure 2: Serous tubal intraepithelial carcinoma. (A \& B) H \& E 10X \& 20X. (C) p53 mutant expression (20X). (D) Proliferatioin index (Ki-67) high in the areas of serous tubal intrepithelial carcinoma, 20X.

HGSC of ovaries had molecular details more comparable to normal fallopian tube epithelium in comparison to ovarian surface epithelium or peritoneum. ${ }^{5}$ Towards the end of $20^{\text {th }}$ Century, fallopian tube epithelium emerged as a possible primary origin of $\mathrm{HGSC}^{1}{ }^{1}$ Before the detailed description of STICS, limited sampling of unremarkable fallopian tubes was performed. Now, authorities in the field recommend sectioning and extensive examination of the fimbria protocol (SEE-Fim). Diagnosis of STIC primarily relies on the sampling of fallopian tube. Although criteria for diagnosing STIC have been proposed, there is still significant interobserver disagreement. ${ }^{6}$ In certain scenarios, frequency of STIC is difficult to determine, like in cases of metastases involving fallopian tube and mimicking STIC, interobserver variability, sampling or effacement offallopian tube by large carcinomas. ${ }^{2}$
Gao et al. performed study in 2013, which included 107 cases of HGSC; and STIC was detected in $80 \%$ of them. They found STIC as a precursor lesion for major bulk of HGSC of fallopian tubes, ovaries and peritoneum. ${ }^{7}$ Carcinomas of gynecological origin other than HGSC and various benign conditions of female genital tract did not show coexistent STICs. ${ }^{8}$

Although all cases of STIC do not progress to HGSC, they are still strongly believed to be a high risk factor for the development of HGSC. Other possible cellular events, which likely cause HGSC, need to be further investigated. ${ }^{1}$ STICs are found in around $50 \%$ of advanced HGSCs. Data suggests that molecular features of HGSCs with and without associated STIC lesions are mostly shared, indicating a common biologic origin, likely to be the fimbriated end of fallopian tube among all cases. ${ }^{5}$

Various ovarian carcinoma-related gene alterations, including TP53, BRCA1 and BRCA2, were also detected in STIC. Some studies have identified seven years gap between the detection of STIC and development of ovarian carcinoma, followed by metastases. ${ }^{9}$ Classical cases of STIC, closely simulate HGSC on microscopy, are associated with HGSC and exhibit genomic instability like HGSC. STICs and p53 signatures are thought as initial events in the progression of HGSC. ${ }^{1}$

Detection of aberrant p53 expression by IHC is a surrogate marker for TP53 mutation. It shows two patterns by IHC, which also accurately predict the type of TP53 mutation. Diffuse positive (overexpression) is due to TP53 missense mutations and diffuse negative (complete absence) results from TP53 nonsense mutations. ${ }^{10}$

STICs have been detected in around $60 \%$ cases of sporadic HGSC. Prophylactic bilateral salpingo-oophorectomy (BSO) specimens of BRCA carriers also show STICs in 10-15\% cases, which also support their primary origin rather than secondary involvement from ovarian HGSC. ${ }^{11}$

In around $40 \%$ cases of HGSC, STIC is not found even after extensive sampling of fallopian tubes. In these cases, proposed theory is the implantation of fimbriated end tubal epithelium onto the ovarian surface during various injuries to ovarian surface like ovulation. Microenvironment of ovary, followed by genetic aberrations, results in malignant transformation. ${ }^{11}$

In conclusion, this case highlights the potential precursor role of STIC in the carcinogenesis of HGSC of ovaries. A meticulous search should always be carried out to find this lesion in cases of HGSCs of ovaries.

\section{PATIENT'S CONSENT:}

Notapplicable.

\section{CONFLICT OF INTEREST:}

The author declared no conflict of interest.

\section{AUTHOR'S CONTRIBUTION:}

$\mathrm{HT}$ : Conception of idea, writing, formatting and finalisation of draft. 


\section{REFERENCES}

1. Kim J, Park EY, Kim O, Schilder JM, Coffey DM, Cho CH, et al. Cell origins of high-grade serous ovarian cancer. Cancers (Basel) 2018; 10(11):433. doi: 10.3390/cancers10110433.

2. Samimi G, Trabert B, Geczik AM, Duggan MA, Sherman ME. Population frequency of serous tubal intraepithelial carcinoma (stic) in clinical practice using see-fim protocol. JNCl Cancer Spectr 2018; 2(4):pky06. doi: 10.1093/jncics/pky061.

3. Lisio MA, Fu L, Goyeneche A, Gao Z, Telleria C. High-grade serous ovarian cancer: Basic sciences, clinical and therapeutic standpoints. Int J Mol Sci 2019; 20(4):E952. doi: 10.3390/ijms20040952.

4. Momenimovahed Z, Tiznobaik A, Taheri S, Salehiniya H. Ovarian cancer in the world: Epidemiology and risk factors. Int J Womens Health 2019; 11:287-99. doi: 10.2147/JWH.S197604.

5. Ducie J, Dao F, Considine M, Olvera N, Shaw PA, Kurman R, et al. Molecular analysis of high-grade serous ovarian carcinoma with and without associated serous tubal intraepithelial carcinoma. Nat Commun 2017; 8(1):990. doi: 10.1038/s41467-017-01217-9.

6. Samimi G, Trabert B, Duggan MA, Robinson JL, Coa KI, Waibel $E$, et al. Processing of fallopian tube, ovary, and endometrial surgical pathology specimens: A survey of U.S. laboratory practices. Gynecol Oncol 2018; 148(3):515-20. doi: 10.1016/j.ygyno.2018.01.016.

7. Gao FF, Bhargava R, Yang H, Li Z, Zhao C. Clinicopathologic study of serous tubal intraepithelial carcinoma with invasive carcinoma: is serous tubal intraepithelial carcinoma a reliable feature for determining the organ of origin? Hum Pathol 2013; 44(8):1534-43. doi: 10.1016/ j.humpath.2012.12.007.

8. Li HX, Lu ZH, Shen K, Cheng WJ, Malpica A, Zhang J, et al. Advances in serous tubal intraepithelial carcinoma: Correlation with high grade serous carcinoma and ovarian carcinogenesis. Int J Clin Exp Pathol 2014; 7(3):848-57.

9. Labidi Galy SI, Papp E, Hallberg D, Niknafs N, Adleff V, Noe $M$, et al. High grade serous ovarian carcinomas originate in the fallopian tube. Nat Commun 2017; 8(1):1093. doi: 10.1038/s41467-017-00962-1.

10. Hatano $Y$, Hatano K, Tamada M, Morishige KI, Tomita $\mathrm{H}$, Yanai $\mathrm{H}$, et al. A comprehensive review of ovarian serous carcinoma. Adv Anat Pathol. 2019; 26(5):329-39. doi: 10.1097/PAP.0000000000000243.

11. Aggarwal IM, Lim YH, Lim TYK. The fallopian tube as the origin of non-uterine pelvic high-grade serous carcinoma. Obstetrician Gynaecologist 2016; 18(2):143-52. doi.org/ 10.1111/tog.12258. 